\title{
ORIGINAL RESEARCH \\ Carotid Artery Plaque Classification: Does Contrast Enhancement Play a Significant Role?
}

L. Saba

M. Piga

E. Raz

D. Farina

R. Montisci

BACKGROUND AND PURPOSE: Previous articles have demonstrated that carotid artery plaques may have enhancement after administration of contrast material. The purpose of this study was to evaluate the effect of enhancement in carotid artery classification.

MATERIALS AND METHODS: Three hundred eighty consecutive patients (235 men, 145 women; median age 64, age range 32-87 years) were analyzed using a multidetector row CT scanner. Examinations were performed before and after $\mathrm{CM}$ administration. Carotid artery plaques with a attenuation value $<60 \mathrm{HU}$ were considered fatty, those from 60-130 HU were considered mixed, and those $>130 \mathrm{HU}$ were considered calcified. $\chi^{2}$ tests, Student $t$ tests, and Cohen analyses were performed.

RESULTS: Before CM administration, we observed 226 calcified, 175 mixed, and 206 fatty plaques; after CM administration, 229 calcified, 213 mixed, and 165 fatty plaques were observed. A statistically significant difference between these 2 groups was observed $(P=.016)$. We found that $19.9 \%$ of fatty plaques become mixed $(n=41)$, whereas $1.7 \%$ of the mixed plaques become calcified $(n=3)$. All calcified plaques remained in the same category. Fatty plaques that changed type showed a larger enhancement compared with those that remained in the same class $(P=.001)$. Cohen analyses showed very good agreement between observers before $(\kappa=0.834)$ and after contrast material administration $(\kappa=0.86)$.

CONCLUSIONS: The results of this study indicate that the carotid artery plaques (fatty and mixed) significantly change according to whether analysis is performed before or after administration of contrast material.

ABBREVIATIONS: $\mathrm{CM}=$ contrast medium; $\mathrm{CPE}=$ contrast plaque enhancement; $\mathrm{HU}=$ Hounsfield unit; MDCTA = multidetector row CT angiography; US = ultrasound

A herosclerosis of the carotid artery represents 1 of the major causes of cerebrovascular disease in the Western world. ${ }^{1}$ Several recent investigations tried to identify those parameters associated with an increased atherosclerotic risk to identify a specific therapeutic approach and reduce the risk of cerebrovascular events. ${ }^{1}$

The degree of luminal stenosis caused by atherosclerotic plaque in the carotid artery is considered an important risk factor of stroke, and the percentage of luminal stenosis degree is used for the choice of the therapeutic approach. ${ }^{2-4}$ However, the severity of carotid stenosis is no longer considered sufficient for identifying patients at high risk for developing an acute cerebrovascular event. The identification of unstable plaque features in vivo may contribute to risk stratification of patients with asymptomatic and moderately severe symptomatic carotid stenosis.

Using CT, several authors ${ }^{5-7}$ differentiated carotid artery plaques into 3 categories: fatty, mixed, and calcified. In this classification, plaques with an attenuation value $<60 \mathrm{HU}$ were considered fatty (soft) plaques, those with a value between 60 and $129 \mathrm{HU}$ were considered mixed, and those with a value $>130 \mathrm{HU}$ were considered calcified. The type of plaque was

Received December 6, 2011; accepted after revision January 23, 2012.

From the Departments of Radiology (L.S., D.F.), Nuclear Medicine (M.P.), and Vascular Surgery (R.M.), Azienda Ospedaliero Universitaria, Cagliari, Italy; Department of Radiology (E.R.), New York University Langone Medical Center, New York, New York.

Please address correspondence to Luca Saba, MD, Department of Radiology, Azienda Ospedaliero Universitaria, di Cagliari - Polo di Monserrato, s.s. 554 Monserrato (Cagliari) 09045, Italy; e-mail: lucasaba@tiscali.it

http://dx.doi.org/10.3174/ajnr.A3073 associated with the presence or absence $e^{8,9}$ of cerebrovascular symptoms, strengthening the concept that the composition of plaque plays a fundamental role in the development of stroke/TIA.

Recently, Saba and Mallarini ${ }^{10}$ demonstrated that carotid artery plaques, when studied with CT, can show contrast enhancement after the administration of contrast medium and that the entity of CPE is associated with the presence of cerebrovascular symptoms.

We hypothesize that CPE may play a role in the HU plaque value; therefore, the purpose of this study was to evaluate the effect of enhancement in the classification of carotid artery plaques.

\section{Materials and Methods}

\section{Study Design and Patient Population}

At our institute, a carotid artery data base was created from retrospective case material, from 2004 onward, that includes all CTA examinations of carotid arteries performed at the institute.

Based on a power calculation (type I error, $\alpha=.01$; type II error, $\beta=.01$; difference $=.05$ ), we estimated that a sample size of at least 400 carotid arteries would be sufficient to evaluate the effect of enhancement in carotid artery plaque classification. Therefore, we searched the data base and found 380 consecutive patients ( $235 \mathrm{men}$; 145 women; median age 64 , age range $32-87$ years) who were examined with MDCTA from May 2006 to February 2011. Part of this population was used in previous studies. ${ }^{10}$ In our institute, CTA was performed when there was a sonography (US) examination that showed pathologic stenosis ( $50 \%$ of stenosis, according to NASCET 

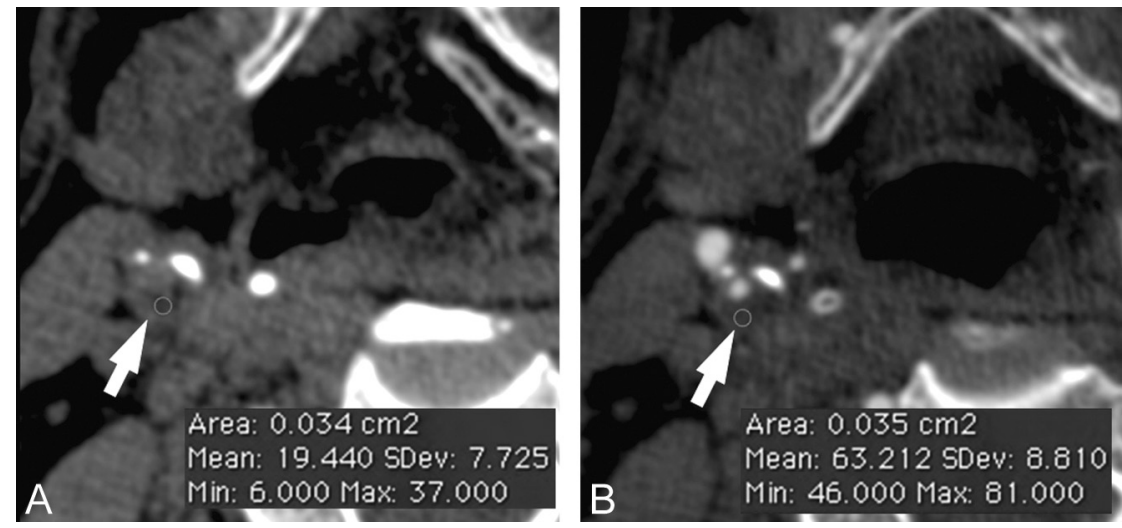

Fig 1. Plaque analysis. In panel $A$, the basal phase is given, whereas panel $B$ is the phase after administration of contrast material. The white arrows indicate the carotid artery plaque, whereas the red circle is the region of interest.

criteria) and/or plaque alteration (presence of heterogeneous plaque, an irregular surface, intraplaque hemorrhage, and/or the presence of ulceration in the plaque). MDCTA was also performed when the US could not provide adequate information about the degree of stenosis and plaque type. Elevated renal function tests or known allergy to iodinated contrast material were considered as exclusion criteria for the MDCTA examination. In our hospital, carotid arteries of asymptomatic patients who underwent cardiac interventions for coronary artery disease, aortic interventions, and lower leg artery surgery, and diabetics who were $>50$ years old, were also studied.

Institutional review board approval was obtained and this work was conducted in accordance with the guidelines of our institution's research committee. Because of the retrospective nature of this analysis, the institutional review board waived the need for ethics committee review.

\section{MDCTA Technique}

All patients underwent MDCTA of the supra-aortic vessels using 2 different CT scanners-a 40 multidetector row CT system (Somatom Sensation; Siemens, Erlangen, Germany) and a 16 multidetector row CT system (Brilliance; Philips, Best, the Netherlands)—using a technique previously described.

In our protocol for the analysis of carotid arteries, a noncontrast scan was performed and was followed by the angiographic phase. The angiographic phase was obtained by injecting $80-90 \mathrm{~mL}$ of contrast medium (Ultravist 370; Schering, Berlin, Germany) into a cubital vein, using a power injector at a flow rate of $5 \mathrm{~mL} / \mathrm{s}$ and an 18-gauge intravenous catheter. A bolus tracking technique was used to calculate the correct timing of the scan. Scans were performed in the caudocranial direction. CT technical parameters included section thickness, $1 \mathrm{~mm}$; interval, $0.5 \mathrm{~mm}$; matrix, $512 \times 512$; FOV , $14-19 \mathrm{~cm}$; mAs, 180-200; and kV, 120-140. Angiographic acquisition included the carotid siphon. None of the patients included in the study had a medical history of cardiac output failure.

\section{Carotid Image Quality and Artifact Evaluation}

Two radiologists (7 and 11 years of experience in MDCTA of the supra-aortic vessel) were asked to evaluate, in consensus, the overall image quality on a 5 -point scale, where 5 corresponded to an excellent image quality, 4 to a good image quality, 3 to an adequate image quality, 2 to a marginally acceptable image quality, and 1 to an unacceptable image quality. Patients for whom at least 1 reader rated image quality as 1 or 2 were excluded from this study.

\section{First Plaque Type Analysis (Contrast-Enhanced Phase)}

Two radiologists (7 and 11 years of experience in MDCTA of the supra-aortic vessel) performed all HU measurements, blinded to each other. Window/level settings were usually set at W850:L300, progressing to very wide settings in the case of attenuated calcifications. ${ }^{11}$

In the first plaque type analysis, we assessed only the contrast material dataset. A circular or elliptical region of interest $\left(\geq 2 \mathrm{~mm}^{2}\right)$ in the predominant area of the plaque was used to measure the HU value (Fig 1). Areas showing contamination by contrast material or calcification that did not contribute to the stenosis were avoided. Regions of beam hardening in calcified areas were also excluded. Moreover, a note was made of the corresponding section to identify any differences between the observers in the choice of the plaque along the z-axis.

\section{Second Plaque Type Analysis (Precontrast Phase)}

The observers first selected the precontrast baseline MDCTA dataset and identified the section corresponding to the contrast enhancement (Fig 1). To obtain a correct registration between the 2 datasets, they visually assessed whether the sections corresponded or whether it was necessary to select another section along the z-axis. After this "matching phase," to measure the basal HU value, a region of interest of the same area as that used in the contrast phase was placed in the same position on the plaque.

\section{Plaque-Type Classification and Interobserver Analysis}

As in previous studies, ${ }^{6,12}$ plaques were classified as 1) fatty-plaques with attenuation $<60 \mathrm{HU} ; 2$ ) mixed-plaques with attenuation between 60 and $129 \mathrm{HU}$; and 3) calcified-plaques with attenuation $>130 \mathrm{HU}$. This classification was based on the precontrast scan.

\section{Statistical Analysis}

To test the differences between plaque classification before and after administration of contrast material, a $\chi^{2} 3 \times 2$ test for differences was used. To perform the analysis, the mean values between the 2 observers were considered. A Student $t$ test for independent samples was used to compare the plaque enhancement in fatty plaques that did not change their category to mixed type versus the plaque enhancement in those fatty plaques that changed their type. Interobserver reliability for plaque classification was calculated using the Cohen $\kappa$ test. The following interpretation of the $\kappa$ statistic was used: $0-0.20=$ poor agreement; $0.21-0.40=$ fair agreement; $0.41-0.60=$ moderate agreement; $0.61-0.80=$ good agreement; and $0.81-1=$ very good 

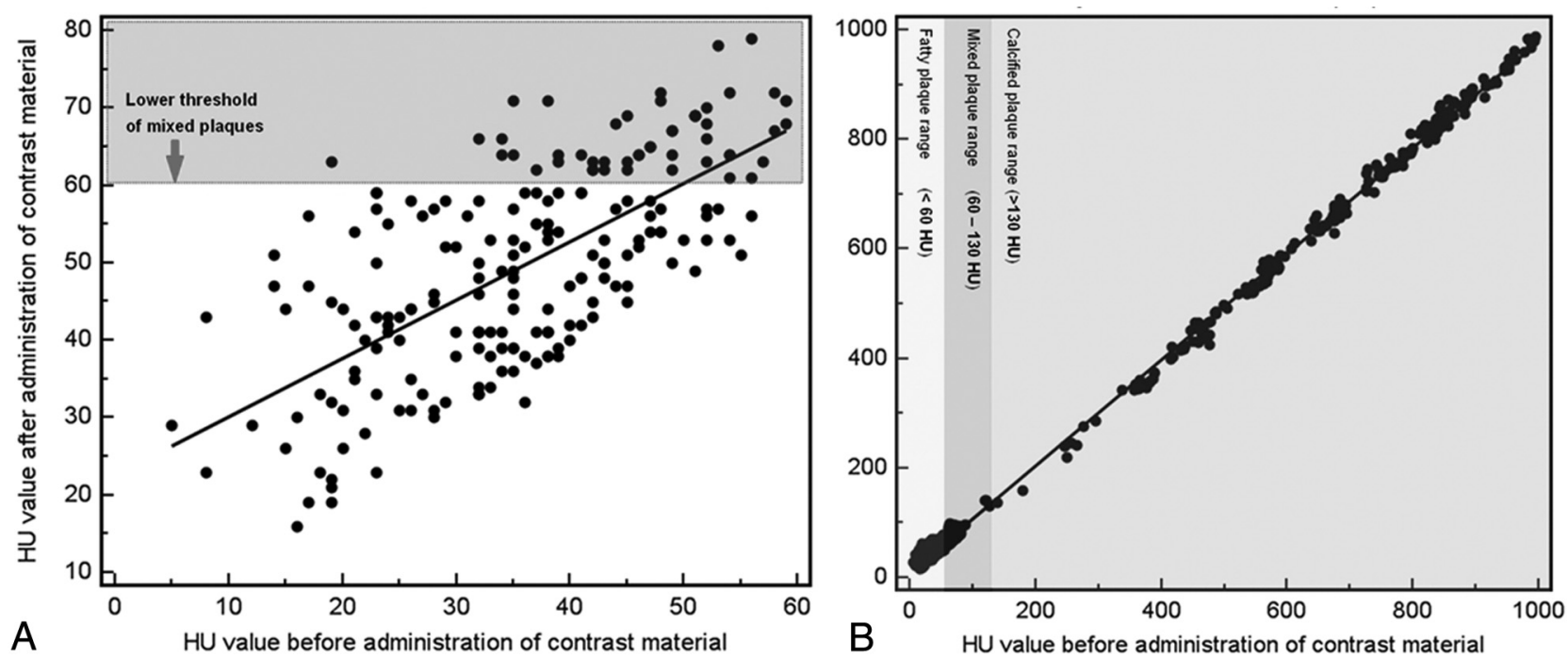

Fig 2. Scatterplots with regression lines $(A, B)$ that show the effect of contrast material administration in plaque classification. In panel $A$, the analysis of $H U$ values before and after administration of contrast material in the 206 plaques that showed $<60 \mathrm{HU}$ in the unenhanced acquisition is given, whereas in panel $B$, the analysis of $\mathrm{HU}$ values before and after administration of contrast material in all the 607 plaques is given.

agreement. To produce these data, R software (www.r-project.org) was used. A $P$ value $<.05$ was regarded as indicating statistical significance.

\section{Results}

\section{Effect of Enhancement in the Plaque Classification}

Sixteen carotid arteries were excluded from the analysis because the image quality was rated $\leq 2$. Another 137 carotid arteries were excluded because no measurable plaque was detected (plaque $\leq 2 \mathrm{~mm}$ ). Therefore, the total number of carotid artery plaques analyzed in this study was 607 . In the basal acquisition, 226 calcified plaques, 175 mixed plaques, and 206 fatty plaques were found, whereas 229 calcified plaques, 213 mixed plaques, and 165 fatty plaques were found after the administration of contrast material.

A statistically significant difference between the groups was observed $(P=.016)$. We observed that $19.9 \%$ of fatty plaques became mixed $(n=41)$, whereas $1.7 \%$ of the mixed plaques became calcified $(n=3)$. All calcified plaques remained in the same category. In Fig 2, scatterplots with a regression line for the $\mathrm{HU}$ value of the plaques before and after administration of contrast material is given to show the magnitude enhancement of the plaques.

The contrast plaque enhancement in fatty plaques that did not change category to the mixed type versus the plaque enhancement of fatty plaques that changed category was compared and we found a statistically significant difference $(P$ value $=.001 ;$ Fig 3$)$.

\section{Interobserver Analysis}

In the basal acquisition, the interobserver agreement in the plaque analysis classification was very good, with a $\kappa$ value of 0.834 ( $95 \%$ CI, 0.797 to 0.872 ); in the arterial acquisition, the interobserver agreement was also very good, with a $\kappa$ value of 0.86 (95\% CI, 0.825 to 0.895$)$.

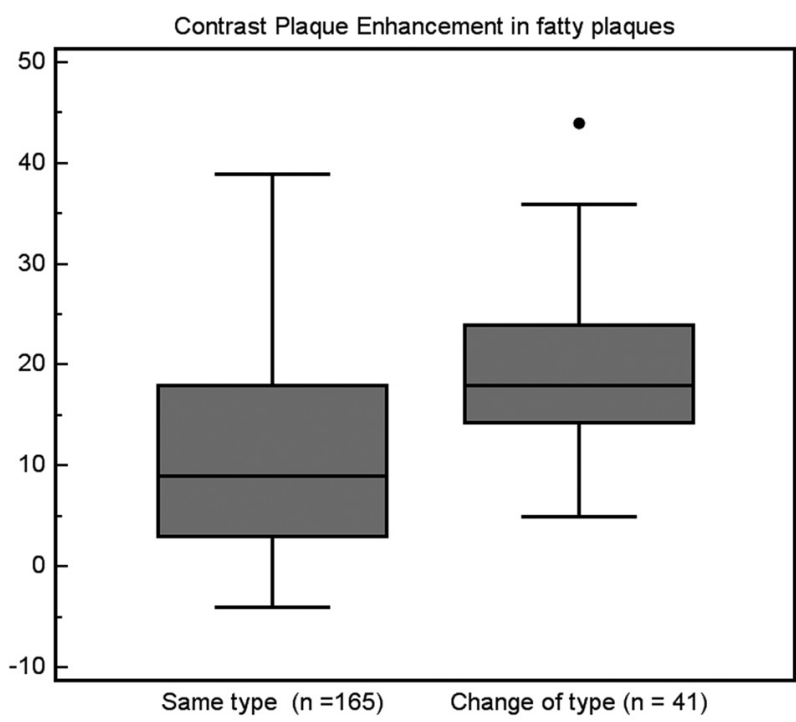

Fig 3. Comparison of the CPE between fatty plaques that did not change after $\mathrm{CM}$ and fatty plaques that changed their type to mixed after CM administration.

\section{Discussion}

Previous studies demonstrated that the degree of luminal stenosis alone is insufficient for predicting carotid plaque vulnerability. ${ }^{13-15}$ Thus, the concept of "vulnerable plaque" has been introduced and authors have suggested that the type of plaque identified using MDCTA can be associated with an increased or reduced risk of experiencing cerebrovascular events. ${ }^{8,12}$ All of these articles analyzed and categorized the carotid artery plaque after the administration of contrast material; however, Saba et $\mathrm{al}^{10}$ recently showed that carotid artery plaques can exhibit contrast enhancement and that the entity of carotid plaque enhancement is associated with an increased risk of cerebrovascular events. Therefore, we hypothesized that the administration of contrast material has an effect in carotid artery plaque classification (because some plaques show enhancement and others do not), and our purpose was to eval- 
uate if this effect is significant from a statistical point of view. In other words, in this article, the focus was to assess whether the use of contrast enhancement modifies the categories within which carotid plaques are placed.

In the analysis of the population, 8 patients (16 carotid arteries) were excluded because of poor image quality (image quality was $\leq 2)$. The prevalence is quite low (2.2\%) and is concordant with previous data reported in the literature. ${ }^{7}$ In these 8 patients, poor image quality was caused by dental hardware artifacts $(n=6)$ and patient movement $(n=2)$.

In the remaining 372 patients (744 carotid arteries), no measurable carotid plaque was detected in 137 carotid arteries (plaque $\leq 2 \mathrm{~mm}^{2}$ ). This does not mean that there was no plaque in these 137 carotid arteries but simply that the plaque was not big enough to reach our cutoff. Therefore, the total number of studied carotid artery plaques was 607 .

After administration of contrast material, 229 calcified plaques, 213 mixed plaques, and 165 fatty plaques were found, whereas 226 calcified plaques, 175 mixed plaques, and 206 fatty plaques were found in the basal acquisition. When we performed the $\chi^{2} 3 \times 2$ test to evaluate the difference between basal and contrast material scans, a statistically significant difference was found between the 2 groups $(P=.016)$. This result suggests that the contrast material may play a significant role in the classification of the type of plaque. In our opinion, the conceptual implication of this result is important: After administration of contrast material, the plaque $\mathrm{HU}$ value does not simply reflect the plaque type (histologic composition) but also other effects like carotid plaque enhancement (namely, the entity of the contrast material that goes within the plaque itself). The direct consequence is that to identify the plaque type as plaque composition, the radiologist should evaluate only the basal acquisition, whereas considering the plaque after administration of contrast material reflects the plaque composition and the plaque enhancement.

In our population, we observed that $19.9 \%$ of fatty plaques become mixed $(n=41)$, whereas $1.7 \%$ of the mixed plaques enter the calcified group $(n=3)$. All calcified plaques remained in the same category. After administration of contrast material, the most affected plaques are the fatty plaques. This fact should be considered because this type of plaque is considered the type that is most frequently associated with the occurrence of cerebrovascular events. ${ }^{8}$ Moreover, we compared the contrast plaque enhancement in fatty plaques that did not change to the mixed category versus the plaque enhancement in fatty plaques that did change categories and found that a statistically significant difference was present $(P$ value $=.001$; Fig 3 ). These findings suggest that some types of fatty plaques may be more vascular compared with other fatty plaques, and this difference may explained by the fact that within the so-called fatty-type plaques, different subtypes of histologic components, like lipid core or intraplaque hemorrhage, are included. ${ }^{6}$ Another hypothesis we can suggest is that some particular condition of the plaque (eg, fissuration of the fibrous cap, ulcerations) can create a breach of the bloodstream into the plaque, with consequent increase in the contrast plaque enhancement.
In this study there are some limitations: one possible objection to our study could be that this is a retrospective analysis, which may introduce bias in the data selection; however, we used data standardization, so the variability in the retrospective analysis should be reduced. A second objection could be the lack of a surgical reference standard. The lack of a reference standard does not allow for the determination of the histologic composition of the plaque; however, our purpose was not to see the concordance between HU values of the plaque in basal scans/after contrast medium scans and histologic composition but to evaluate the effect of enhancement in the carotid artery classification.

\section{Conclusions}

The results of this study indicate that carotid artery plaque type (fatty and mixed) significantly changes according to whether the analysis is performed before or after administration of contrast material. Therefore, classification of the plaque type should be performed from the basal scans.

\section{References}

1. von Sarnowski B, Lüdemann J, Völzke H, et al. Common carotid intima-media thickness and Framingham Risk Score predict incident carotid atherosclerotic plaque formation: longitudinal results from the study of health in Pomerania. Stroke 2010;41:2375-77

2. Rothwell PM, Eliasziw M, Gutnikov SA, et al. Analysis of pooled data from the randomised controlled trials of endarterectomy for symptomatic carotid stenosis. Lancet 2003;361:107-16

3. Rothwell PM, Warlow CP. Prediction of benefit from carotid endarterectomy in individual patients: a risk-modelling study. European Carotid Surgery Trialists' Collaborative Group. Lancet 1999;353:2105-10

4. North American Symptomatic Carotid Endarterectomy Trial Collaborators. Beneficial effect of carotid endarterectomy in symptomatic patients with high grade stenosis. N Engl J Med 1991;325:445-53

5. de Weert TT, Ouhlous M, Zondervan PE, et al. In vitro characterization of atherosclerotic carotid plaque with multidetector computed tomography and histopathological correlation. Eur Radiol 2005;15:1906-14

6. de Weert TT, Ouhlous M, Meijering E, et al. In vivo characterization and quantification of atherosclerotic carotid plaque components with multidetector computed tomography and histopathological correlation. Arterioscler Thromb Vasc Biol 2006;26:2366-72

7. de Weert TT, de Monyé C, Meijering E, et al. Assessment of atherosclerotic carotid plaque volume with multidetector computed tomography angiography. Int J Cardiovasc Imaging 2008;24:751-59

8. Saba L, Montisci R, Sanfilippo R, et al. Multidetector row CT of the brain and carotid artery: a correlative analysis. Clin Radiol 2009;64:767-78

9. Nandalur KR, Baskurt E, Hagspiel KD, et al. Calcified carotid atherosclerotic plaque is associated less with ischemic symptoms than is noncalcified plaque on MDCT. AJR Am J Roentgenol 2005; 184:295-98

10. Saba L, Mallarini G. Carotid plaque enhancement and symptom correlations: an evaluation by using multidetector row CT angiography. AJNR Am J Neuroradiol 2011;32:1919-25

11. Saba L, Mallarini G. MDCTA in the study of carotid plaque stenosis degree: evaluation of inter-observer agreement. AJR Am J Roentgenol 2008;190: W41-46

12. Rozie S, de Weert TT, de Monyè C, et al. Atherosclerotic plaque volume and composition in symptomatic carotid arteries assessed with multidetector CT angiography; relationship with severity of stenosis and cardiovascular risk factors. Eur Radiol 2009;19:2294-301

13. Wasserman BA, Wityk RJ, Trout HH, et al. Looking beyond the lumen with MRI. Stroke 2005;36:2504-13

14. Lovett JK, Gallagher PJ, Hands LJ, et al. Histological correlates of carotid plaque surface morphology on lumen contrast imaging. Circulation 2004;110:2190-97

15. Lovett JK, Gallagher PJ, Rothwell PM. Reproducibility of histological assessment of carotid plaque: implications for studies of carotid imaging. Cerebrovasc Dis 2004;18:117-23 